\title{
LETTER \\ Reconstitution of Potential Function by Power Spectra of Trajectories in Nonlinear Dynamical Systems
}

\author{
Masataka MINAMI $^{\dagger \mathrm{a})}$, Student Member and Takashi HIKIHARA ${ }^{\dagger \mathrm{b})}$, Member
}

\begin{abstract}
SUMMARY Phase structure of nonlinear dynamical system is governed by the vector field and decides the trajectories. Accordingly, the power spectra of trajectories include the structural field effect on the phase space. In this paper, we develop a method for analyzing phase structure using power spectra of trajectories and reconstitute a potential function in the system.

key words: power spectra, potential function, reconstitution, nonlinear dynamical systems
\end{abstract}

\section{Introduction}

State variables in a nonlinear dynamical system temporally show the motions similar to a rolling ball in the potential well. The potential well is characterized by equilibrium points and vector flow in the phase space around the points. Therefore, the phase space analysis is of basic importance for understanding nonlinear dynamical systems in science and controlling systems in engineering. There are many analytical and numerical methods for analysis of phase space structures: averaging, perturbation, Poincaré map [1], cellto-cell mapping [2], and so forth. Trajectories are governed by the geometry of potential function on the phase space in both short and long distance. Therefore, it is substantial to develop a methodology and tools for reconstitution of the potential function for unknown system. In this paper we develop a method for analyzing the phase space structures using the power spectra of trajectories [3] and reconstitute a potential function in the system.

Many groups of researchers have worked at the intersection of phase space analysis and spectrum analysis. The spectrum of dynamical systems has been extensively studied since the Koopman's pioneering work [4]. These notions of spectrum can be linked with the terms of ergodic theory in dynamical systems [5]. In particular, the analyses based on operators are proposed for extracting invariant measures and statistics values [6]-[8]. The analysis for Arnold web resonances are proposed to an experimentally accessible atomic system that may well form a paradigm for phenomena in several degrees of freedom [9]. The method fundamentally focuses on power spectrum distribution in parameter space [3]. In [10] the authors performed a time-frequency analysis of Hamiltonian systems based on a ridge extraction from

\footnotetext{
Manuscript received September 2, 2011.

${ }^{\dagger}$ The authors are with the Department of Electrical Engineering, Kyoto University, Kyoto-shi, 615-8510 Japan.

a)E-mail:minami@kuee.kyoto-u.ac.jp

b) E-mail: hikihara.takashi.2n@kyoto-u.ac.jp

DOI: 10.1587/transfun.E95.A.613
}

a wavelet decomposition of a single-trajectory coordinate. The ridge pattern can reveal the phase space structures and give a characterization of weak or strong chaos. In [11], [12] the authors develop a method for visualization of dynamical systems with a smooth invariant measure. The method uses time average along trajectories of a set of functions defined on the phase space, which are called observables, and enables the detection of ergodic invariant sets. Also, in [8], [13] the authors present a visualization of periodic sets in phase space using the concept of harmonic time average found in [14]. These methods are associated with spectrum properties of the Koopman operator [14]. In [15] the authors develop a numerical method for detection of almostinvariant sets [16], [17], which are an extension of invariant sets, using the transfer operator approach. The method is applied to analysis of coherent structures arising in transport and mixing of fluid flows [18]. They show that the structure of almost-invariant sets is associated with the eigen structure of the transfer operator, namely, the Perron-Frobenius operator.

This paper is organized as follows. Section 2 introduces and assumes a nonlinear dynamical system in this paper. We focus on the relationship between the potential function and the power spectra of finite-time trajectories, confirmed in Sect. 3. Section 4 addresses a method for reconstituting the potential function in conservative systems. It will be the basis of the following discussion. Section 5 applies the method to a conservative, damped, and forced system. Section 6 remarks the results of this paper.

\section{System Setting}

This section introduces a nonlinear dynamical system confirmed in this paper. It is assumed that state variables in the system show one-degree-of-freedom motions. We consider the system having one-degree-of-freedom so as to visualize our results in the later sections, and to understand a potential function.

A potential function leads restoring force in the nonlinear dynamical system. A continuous-time dynamical system on phase space $X$, which is assumed 2-dimensional smooth manifold, is described by

$$
\frac{\mathrm{d} x}{\mathrm{~d} t}=y, \quad \frac{\mathrm{d} y}{\mathrm{~d} t}=-\varepsilon k y-\frac{\mathrm{d} U(x)}{\mathrm{d} x}+\varepsilon B \cos t,
$$

where $[x, y]^{\mathrm{T}} \in X, U(x)$ is a smooth nonlinear function, $-\varepsilon k y$ denotes the damping force term, $-\mathrm{d} U(x) / \mathrm{d} x$ the restor- 
ing force term, and $\varepsilon B \cos t$ the external force term. $\varepsilon$ is relatively small enough. If $-\varepsilon k y$ and $\varepsilon B \cos t$ are negligible, then Eq. (1) becomes the following conservative system:

$$
\frac{\mathrm{d}^{2} x}{\mathrm{~d} t^{2}}=-\frac{\mathrm{d} U(x)}{\mathrm{d} x} .
$$

The shape of potential function is considered for the system. It is assumed that the potential function is given by $U(x)=\sum_{i=0}^{n} a_{i} x^{i}$, where $n$ is a finite integer and $a_{i} \in \mathbb{R}(i=$ $0,1, \cdots, n)$ is a constant coefficient. In addition, bounded trajectories are assumed in order to focus on a power spectrum of local and global oscillations in this paper. Then $n$ is even and $a_{n}>0$.

\section{Power Spectra of Trajectory and Its Calculation Set- ting}

The notion of power spectrum is confirmed for a time series, which originates the trajectory, generated by the dynamical system given by Eq. (1). The power spectrum describes the power distribution of a time series in frequency domain [19]. Here we consider the power spectrum of an observable time series $\psi(t)$. The observable time series implies a physically-measured time series generated from the dynamical system given by Eq. (1). For the time series $\psi(t)$, the power spectrum is defined as the Fourier transform of the auto-correlation function [19], [20]:

$$
S_{\psi}(\omega) \triangleq \int_{-\infty}^{\infty}\left[\frac{1}{t_{1}-t_{0}} \int_{t_{0}}^{t_{1}} \psi(t) \psi(t+\tau) \mathrm{d} t\right] \mathrm{e}^{-\mathrm{i} \omega \tau} \mathrm{d} \tau
$$

In the following, it is assumed that the power spectrum is well-defined in the nonlinear dynamical system of our interest.

The method for calculating the power spectrum of a finite-time trajectory is described as follows. The power spectrum is generally defined as infinite interval $(-\infty, \infty)$, that is $t_{0} \rightarrow-\infty$ and $t_{1} \rightarrow+\infty$ in Eq. (3). However, it is impossible to calculate the trajectory in the infinite interval. The physically-measured time series is finite data. It is significant to consider the power spectrum of a finitetime trajectory. Then, an augmented trajectory is defined as $[x(t), y(t)]^{\mathrm{T}}$ for $t \in\left[t_{0}, t_{1}\right]$ and $[0,0]^{\mathrm{T}}$ for other.

The idea of a numerical algorithm is described. Trajectory for each initial state is calculated by the integration through the fourth order Runge Kutta algorithm until 120 cycles of the external force. The finite interval is $0 \leq t \leq 2 \pi \times 120$, that is $t_{0}=0$ and $t_{1}=2 \pi \times 120$. The duration has two means. One is that the duration is sufficiently long, and the other is the aliquots of the number 120 . The power spectrum of the solution is estimated by the time step of the fourth order Runge Kutta method: $2 \pi \times 120 /\left(100 \times 2^{14}\right)$ and the number of data for the auto-correlation function of the trajectory is $2^{14}$. As results, Eq. (3) gives the power spectrum of finite-time trajectory. In this paper $x(t)$ in Eq. (1) is regarded as observable time series $\psi(t)$.

\section{Analytical Reconstitution of Potential Function in Conservative System}

This section develops a method for reconstituting a potential function in the conservative system given by Eq. (2). The results of this section will contribute to the basis of the following discussion in Sect. 5.

Trajectories are governed by the geometry of potential function on the phase space in both short and long distance as mentioned above. In short distance, state variables oscillate in the potential well. In other words, the trajectories stay around vicinity of stable sink in Eq. (1). In general, nonlinear dynamical systems are linearized in the vicinity of equilibrium points in order to analyze the characteristics of the points and vector flow related to trajectories. Analysis based on the lowest dimensional component of potential function enables to grasp motions. On the other hand, in long distance, trajectories are governed by highest dimensional component of potential function in Eq. (1) when trajectories have high amplitude. Hence, it is significant to analyze the trajectories based on monomial potential functions. Furthermore, equilibrium point of saddle type forms a potential peak. In the following sections, we will discuss these based on relationship between the potential function and power spectrum.

\subsection{Relationship between Monomial Potential Function and Power Spectrum}

At the beginning of discussion, a method for reconstituting a monomial potential function is introduced. State variables in Eq. (2) periodically oscillate. Thus, $x(t)$ gives the period $T_{1}$ and the fundamental angular frequency $\omega_{1} . T_{1}$ and $\omega_{1}$ are determined by the potential function and the initial state. The correspondence induces a method for reconstituting a potential function.

The low-amplitude and high-amplitude oscillation are dominated by monomial potential functions. Here, we consider $a_{n} x^{n}$ as a potential function and address a method for reconstituting a monomial potential function based on the relationship between initial state and $T_{1}$ or $\omega_{1}$.

Setting an initial state at $x(0)=x_{0}$ and $y(0)=0$. The potential function shows peak at $x_{0}$. The relationship between the period $T_{1}$ and the fundamental angular frequency

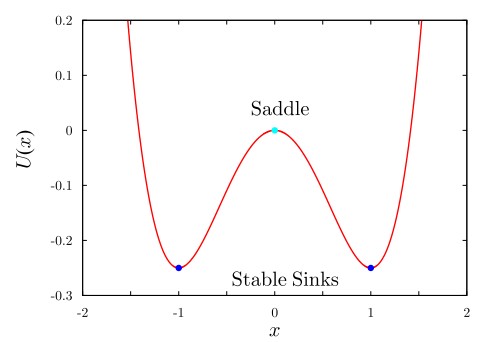

Fig. 1 Potential function of $U(x)=x^{4} / 4-x^{2} / 2$. Saddle exists at $x=0$ and two stable sinks exist at $x= \pm 1$. 


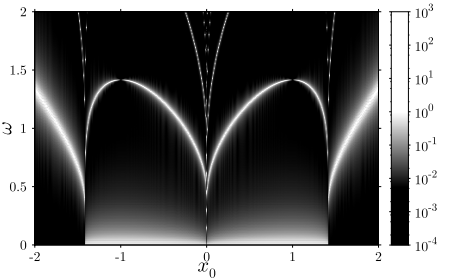

(a) $\varepsilon k=0, \varepsilon B=0$

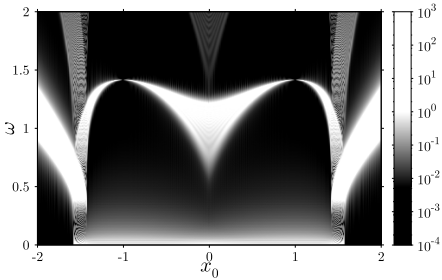

(b) $\varepsilon k=0.001, \varepsilon B=0$

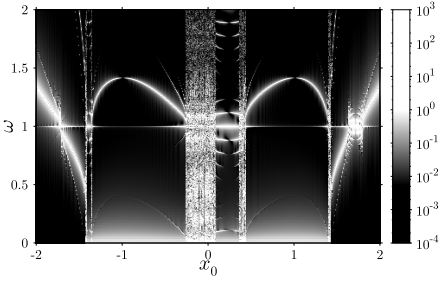

(c) $\varepsilon k=0, \varepsilon B=0.01$

Fig. 2 Decomposition of power spectra in the Eq. (1). The initial state is $x(0)=x_{0}$ and $y(0)=0$.

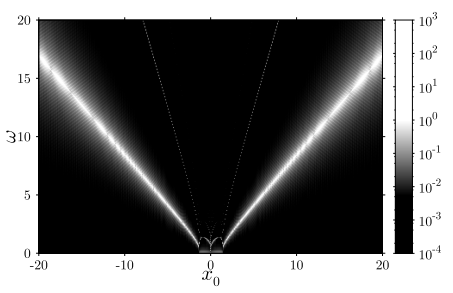

(a) $\varepsilon k=0, \varepsilon B=0$

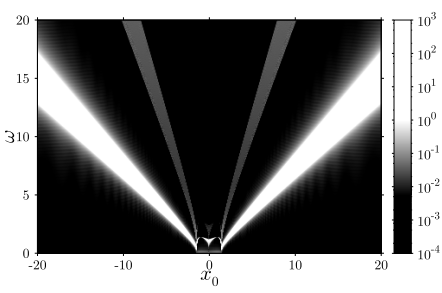

(b) $\varepsilon k=0.001, \varepsilon B=0$

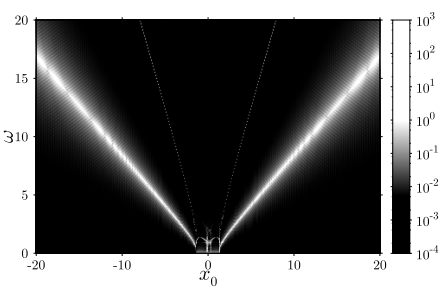

(c) $\varepsilon k=0, \varepsilon B=0.01$

Fig. 3 Decomposition of power spectra in the Eq. (1). The initial state is $x(0)=x_{0}$ and $y(0)=0$ in global range.

$\omega_{1}$ holds $\omega_{1}=2 \pi / T_{1}$. Then, $\omega_{1}$ is estimated by [21], [22]:

$$
\omega_{1}=\sqrt{\frac{a_{n} \pi}{2}} n \frac{\Gamma\left(\frac{1}{n}+\frac{1}{2}\right)}{\Gamma\left(\frac{1}{n}\right)}\left|x_{0}\right|^{\frac{n}{2}-1},
$$

where $\Gamma(x)$ is the Gamma function, which is defined as $\int_{0}^{\infty} t^{x-1} \mathrm{e}^{-t} \mathrm{~d} t$. At $n=2$, Eq. (4) becomes a linearized eigen value of stable sink. Power spectrum of the trajectory analytically signifies $n$ and $a_{n}$ through Eq. (4).

\subsection{Motion in Potential Well}

The proposed analysis is the power spectrum analysis for the trajectories originated from vicinity of a saddle point. The trajectory shows oscillation in potential well. Let us consider $U(x)=x^{4} / 4-x^{2} / 2$ as the potential function. The potential function is drawn in Fig. 1. There exist equilibrium points at $x=0$ and $x= \pm 1$. They are a saddle at $x=0$ and stable sinks at $x= \pm 1$.

The linearized eigen values around the saddle are depicted by $\lambda_{1}, \lambda_{2}$. In particular, $\lambda_{1}=1, \lambda_{2}=-1$. In the vicinity of the saddle, the trajectories are governed by the eigen vectors. Setting the initial state at $x(0)=x_{0}$ and $y(0)=0$, the trajectory passes from $x_{0}$ to $x_{1}, x_{0}$ and $x_{1}$ keep the relationship $U\left(x_{0}\right)=U\left(x_{1}\right)$. Then the period $T_{1}$ of the trajectory satisfies

$$
\frac{\lambda_{1} T_{1}}{2}=\ln x_{1}-\ln x_{0}+\delta,
$$

where $\delta$ denotes a linear error term. Hence, the period $T_{1}$ presents the eigen value of the saddle with respect to an unknown potential function $U(x)$.

\section{Applications to the Nonlinear Oscillator}

This section applies the proposed method to the nonlinear oscillator given by Eq. (1). At first, decomposition of power spectra from trajectories is requested for reconstituting potential functions by the method in Sect. 3 .

Figure 2 shows the numerically obtained decomposition of power spectra by Eq. (1) with $U(x)=x^{4} / 4-x^{2} / 2$. The initial state is set at $x(0)=x_{0}, y(0)=0$. The color contour visualizes the power spectra in $\left(x_{0}, \omega\right)$ plane. Figures 2(a), 2(b), and 2(c) correspond to conservative, damped, and forced system owing to setting $\varepsilon k$ and $\varepsilon B$ respectively. Figure 2(a) shows strong power spectra with respect to the fundamental angular frequency $\omega_{1}$. In Fig. 2(b) the power spectra spreads to frequency domain because the trajectories converge to the stable sinks. The spread depends on the convergence time due to the initial state. It is positively explained that the power spectra include the convergent characteristic in finite time. In Fig. 2(c), the power spectra also include the components of the angular frequency by the external excitation set at $\omega=1$. It shows the feature of modulation in the distribution around $\omega=1$. The power spectra of the trajectories from the vicinity of the saddle include several component of the angular frequencies, since the trajectories traverse between two potential wells. The discussion on the details is not the aim of this paper.

In the vicinity of stable sinks at $x_{0}= \pm 1$, the fundamental angular frequency $\omega_{1}$ converges to $\sqrt{2}$ in Fig. 2. Equation (4) and the result lead the lowest dimension of the potential function to $n=2$. The coefficient $a_{2}$ becomes unity. In fact, $U(x)$ is transformed to $U\left(x^{\prime}\right)=x^{\prime 4} / 4+x^{\prime 3}+x^{\prime 2}-1 / 4$ 


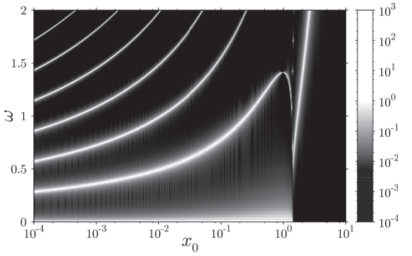

(a) $\varepsilon k=0, \varepsilon B=0$

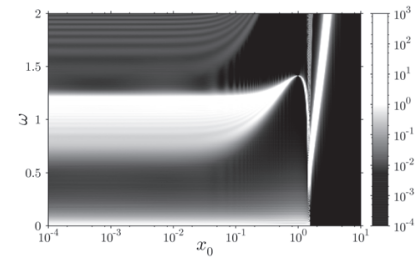

(b) $\varepsilon k=0.001, \varepsilon B=0$
Fig. 4 Decomposition of power spectra in the Eq. (1), where $\varepsilon k=0$ and 0.001 and $\varepsilon B=0$ in the vicinity of saddle at $x=0$.

by $x^{\prime}=x-1 . x^{\prime}$ is a variational displacement around the stable sink at $x=1$. The potential function $U\left(x^{\prime}\right)$ can be approximated as $U\left(x^{\prime}\right)=x^{\prime 2}$ in the vicinity of the stable sink at $x=1$. Therefore, the proposed methods provide to reconstitute the potential function.

The validity of the proposed method must be confirmed in the global range. Figure 3 shows the decomposition of power spectra of trajectories. Figures 3(a) and 3(c) show bright line spectrum so that Eq. (4) leads the highest dimension of the potential function and the coefficient. On the other hand, the power spectra spread in frequency axis in Fig. 3(b), because the trajectories converge to the stable sinks. Thus, the upper edge of bright line corresponds with $\omega_{1}$ in the Eq. (4).

The power spectra is confirmed for the trajectories originated from vicinity of saddle. Figure 4 shows the decomposition of the power spectra by Eq. (1). In Fig. 4(a), strong power spectra show high intention at the fundamental angular frequency $\omega_{1}$ and the harmonic angular frequency $2 \omega_{1}, 3 \omega_{1}, 4 \omega_{1}, 5 \omega_{1}$, and $6 \omega_{1}$. The result is expected by Eq. (5). A little difference of initial state makes a large difference of power spectrum. On the other hand, in Fig. 4(b) the power spectra spread to upper frequency. It is because a damping force decreases a difference of power spectrum by a difference of initial state.

\section{Remarks}

This paper focused on the relationship between power spectra and potential function. The power spectra analytically reconstituted a monomial potential function. In addition, the power spectra presented an eigen values of a saddle in the conservative system. The proposed method was applied to low-dimensional nonlinear dynamical systems. However, our contribution will provide the fundamentals for the case of high-dimensional system. As results, it is clarified that the proposed method has a possibility of reconstituting the potential function in the nonlinear dynamical system. Since the power spectra is closely related to the study of observable time series, the proposed method are expected to find applications in sciences and engineering.

\section{Acknowledgments}

The authors would like to show their acknowledge to
Mr. Takumi Ikenoue for his launch of early research. The authors acknowledge Professor Yoshihiko Susuki for his fruitful comments and discussions. This research was partially supported by the Global COE Program of Kyoto University.

\section{References}

[1] J. Guckenheimer and P. Holmes, Nonlinear Oscillations, Dynamical Systems, and Bifurcations of Vector Fields, ch. 1, Springer-Verlag, 1983.

[2] C.S. Hsu, Cell-to-cell Mapping - A Method of Global Analysis for Nonlinear Systems, Springer-Verlag, 1987.

[3] M.S. Bartlett, "Periodogram analysis and continuous spectra," Biometrika, vol.37, no.1/2, pp.1-16, 1950.

[4] B.O. Koopman, "Hamiltonian systems and transformations in Hilbert space," Proc. National Academy of Sciences of the United States of America, vol.17, no.5, pp.315-318, 1931.

[5] H. Broer and F. Takens, "Mixed spectra and rotational symmetry," Archive for Rational Mechanics and Analysis, vol.124, no.1, pp.1342, 1931.

[6] J. Ding, "The point spectrum of Frobenius-Perrono and Koopman operators," Proc. American Mathematical Society, vol.126, no.5, pp.1355-1361, 1998.

[7] A. Lasota and M.C. Mackey, Chaos, Fractals, and NoiseStochastic Aspects of Dynamics, Second ed., Springer, 1998.

[8] I. Mezić, "Spectral properties of dynamical systems, model reduction and decompositions," Nonlinear Dynamics, vol.41, no.1/3, pp.309-325, 2005.

[9] J.V. Milczewski, G.H.F. Diercksen, and T. Uzer, "Computation of the Arnold's web for hydrogen atom in crossed electric and magnetic fields," Phys. Review Lett., vol.76, no.16, pp.2890-2893, 1996.

[10] C. Chandre, S. Wiggins, and T. Uzer, "Time-frequency analysis of chaotic systems," Physica D, vol.181, no.3/4, pp.171-196, 2003.

[11] I. Mezić and S. Wiggins, "A method for visualization of invariant sets of dynamical systems based on the ergodic partition," CHAOS, vol.9, no.1, pp.213-218, 1999.

[12] Z. Levnajić and I. Mezić, "Ergodic theory and visualization. I. mesochronic plots for visualization of ergodic partition and invariant sets," CHAOS, vol.20, no.033114, pp.1-19, 2010.

[13] Z. Levnajić and I. Mezić, "Ergodic theory and visualization II: Visualization of resonances and periodic sets," pp.1-14 Preprint: arXiv:0808.2182, 2008.

[14] I. Mezić and A. Banaszuk, "Comparison of systems with complex behavior," Physica D, vol.197, no.1/2, pp.101-133, 2004.

[15] G. Froyland and K. Padbergb, "Almost-invariant sets and invariant manifolds - Connecting probabilistic and geometric descriptions of coherent structures in flows," Physica D, vol.238, no.16, pp.1507$1523,2009$.

[16] M. Dellnitz and O. Junge, "Almost invariant sets in Chua's circuit," International Journal of Bifurcation and Chaos in Applied Sciences and Engineering, vol.7, no.11, pp.2475-2485, 1997.

[17] M. Dellnitz and O. Junge, "On the approximation of complicated dynamical behavior," SIAM J. Numerical Analysis, vol.36, no.2, pp.491-515, 1999.

[18] G. Froyland, K. Padbergb, M.H. England, and A.M. Treguier, "Detection of coherent oceanic structures via transfer operators," Phys. Rev. Lett., vol.98, no.22, pp.224503-1-224503-4, 2007.

[19] E.J. Hannan, Time Series Analysis, ch. 3, Chapman \& Hall/CRC, 1967.

[20] A. Papoulis, The Fourier Integral and Its Applications, pp.240-252, McGraw-Hill, 1962.

[21] L.D. Landau and E.M. Lifshitz, Mechanics, third ed., pp.25-29, 7074, Pergamon Press, 1976.

[22] H. Goldstein, Classical Mechanics, second ed., pp.59-64, AddisonWesley, 1980. 